\title{
Dynamic assessment of the tear film muco-aqueous and lipid layers using a novel tear film imager (TFI)
}

Fani Segev 1, 2, Noa Geffen 1, 2, Anat Galor 3, 4, Yoel Cohen ${ }^{5}$, Raanan Gefen ${ }^{5}$, Avner Belkin ${ }^{1}$, Yoel Arieli ${ }^{5,6}$, Shlomi Epshtein ${ }^{5}$, Anat Oren ${ }^{2}$, Alon Harris ${ }^{7}$

1. Ophthalmology, Meir Medical Center, Kfar Sava, Israel.

2. Department of Ophthalmology - Sackler Faculty of Medicine, Tel Aviv University, Tel Aviv, Israel.

3. Ophthalmology, Miami VA Medical Center, Miami, Florida, USA agalor@med.miami.edu.

4. Bascom Palmer Eye Institute, University of Miami, Miami, Florida, USA.

5. R\&D department, AdOM Advanced Optical Technologies, Lod, Israel.

6. Department of Applied Physics, The Jerusalem College of Technology, Jerusalem, Israel.

7. Department of Ophthalmology, Indiana University School of Medicine, Indianapolis, Indiana, USA.

\begin{abstract}
Purpose The objective of the study was to assess a new technology, the tear film imager (TFI), which can dynamically image the muco-aqueous and lipid layers.

Methods Prospective pilot case series of individuals with and without dry eye (DE). Two sequential images were obtained with the TFI. Measurements were assessed for reproducibility and compared with clinically derived DE metrics. Individuals were grouped into DE categories based on signs of DE.

Results 49 patients participated in the study with a mean age of 58.8 years (SD 15.9) and a female majority (69\%). Reproducibility of the muco-aqueous layer thickness (MALT) was excellent $(r=0.88)$. MALT measurements significantly correlated with the Schirmer score $(r=0.31)$. Lipid break up time (LBUT) as measured by the TFI significantly correlated with the clinical measure of tear break up time (TBUT) $(r=0.73)$. MALT and LBUT were significantly thinner and shorter, respectively, in the DE groups (mild-moderate and severe) compared with the control group. When comparing TFI parameters to clinically assessed signs, sensitivity of the device was $87 \%$ and specificity was $88 \%$.
\end{abstract}

Conclusion The TFI is the first machine capable of reproducibly measuring muco-aqueous thickness in human subjects which correlates with Schirmer score. In parallel, it assesses other important aspects of tear film function which correlate with clinician assessed DE metrics.

Keywords: dry eye; imaging; tear breakup; tear film; technology.

This is the author's manuscript of the article published in final edited form as:

Segev, F., Geffen, N., Galor, A., Cohen, Y., Gefen, R., Belkin, A., Arieli, Y., Epshtein, S., Oren, A., \& Harris, A. (2020). Dynamic assessment of the tear film muco-aqueous and lipid layers using a novel tear film imager (TFI). British Journal of Ophthalmology, 104(1), 136-141. https://doi.org/10.1136/bjophthalmol-2018-313379 


\section{INTRODUCTION}

Dry eye (DE) is a common condition that includes a wide range of symptoms (vision and pain related) and signs (evaporative and aqueous deficiency). 1 These clinical features are driven by a multitude of pathophysiological mechanisms (eg, inflammation, hyperosmolarity, nerve dysfunction, meibomian gland disease) that lead to a lack of tear homeostasis. $\stackrel{2}{\mathrm{DE}}$ symptoms are a source of morbidity, affecting both physical and mental functioning. $\frac{3}{}$

Given the many components of DE, different tests have been developed to assess various components of the tear film. Clinical tests are the ones most frequently used and indirectly measure different components of the lacrimal functional unit. For example, tear break up time (TBUT) indirectly measures evaporation and the Schirmer's test indirectly measures tear production. $\stackrel{4}{ }$ These metrics are enhanced by a variety of imaging techniques that capture one or more components of the lacrimal functional unit. For example, LipiView (TearScience, Morrisville, North Carolina, USA) can quantify lipid thickness and provide images of meibomian glands $\frac{5}{5}$; Keratograph $5 \mathrm{M}$ (OCULUS, Arlington, Washington, USA) can quantify TBUT and tear meniscus height and provide images of meibomian glands $\underline{\underline{6}}$ and high-resolution anterior segment optical coherence tomography (HR-OCT) machines can provide cross-sectional images of the precorneal tear film, inferior tear meniscus tear and meibomian glands. $\frac{7-9}{-}$ Limitations with current technologies include quantifying tear parameters in a static manner, as is the case for tear meniscus height, and not having enough resolution to capture muco-aqueous thickness information. This reality makes the assessment of DE cumbersome as the physician must employ several clinical and diagnostic tests to evaluate the tear film.

In the last few years, several attempts have been made to apply spectral interference methods to non-invasively study the thickness of the human tear film. 10 This was demonstrated in vivo in humans with and without a contact lens. $\frac{1011}{1}$ This technology was also applied to study the thinning rate of the tear film with and without emulsion eyedrops. $\frac{12}{}$ However, there is currently no commercially available device that uses spectral interference to measure tear film parameters. 
Recently, a device was developed by AdOM (Israel) that can directly and dynamically measure several aspects of tear physiology with one measurement. The tear film imager (TFI) uses spectral interference technology to image the corneal surface with a large field of view ( $6 \mathrm{~mm}$ diameter) and high lateral resolution. The TFI can measure muco-aqueous layer thickness (MALT) $\frac{13}{3}$ and lipid layer thickness (LLT) and assess for change over time. We herein report the initial results of the TFI device study. The primary goal of this pilot study was to assess safety and measurement reproducibility of the instrument. A secondary goal was to compare clinically assessed DE metrics with machine-assessed metrics.

\section{METHODS}

The study was conducted at the Meir Medical Center in Kfar Saba, Israel. The study was conducted in accordance to the principles of the Declaration of Helsinki. The novel TFI technology used was also approved by the Ministry of Health of Israel.

\section{TFI device}

The TFI is an optical device that illuminates broadband light at wavelengths that range from 450 to $1050 \mathrm{~nm}$ from a small halogen light source. The illumination optics project light towards the corneal front surface at broad angles of -50 to 50 degrees, while ensuring that light level is within the safety limits. The use of very wide angles ensures that the generated image will have large field of view. In contrast, OCT devices use a narrowband at the infrared wavelength and their axial resolution (A-scan resolution) depends on a narrowband interference measurement. As such, while both OCT and TFI technologies use reflected light interference patterns, the hyperspectral TFI analyses wideband reflectance (600 $\mathrm{nm}$ band), while the OCT analyses narrowband reflectance (tens of nanometres with the swept source OCT at most). This fundamental difference allows the TFI to reach single nanometre resolution when assessing the tear film sublayers, while the OCT can at most reach $2-3 \mu \mathrm{m}$ resolution, more than three orders of magnitude apart.

A small custom objective, positioned in the centre of the illumination lens, collects the reflected specular light from the curved corneal surface and creates a flat conjugate 
image at the camera and spectrometer locations. This image is used for capturing and analysing eye movements, blinks and for supporting the auto focus real time operations. The colour information in each pixel of the image is used to determine the interference of light resulting from the impact of the illuminated light with the thin layers of the tear film. Detailed spectrum data is used to analyse the tear film structure at the cornea centre, by comparing the collected spectral data to the anticipated reflectance spectra. The result of this fit yields thickness values for the muco-aqueous and lipid layers several times in each second. Typically, 10-15 values are collected per second during a measurement period of $40 \mathrm{~s}$.

The frequent measurements of the MALT and LLT construct a time series for each layer thickness parameter. The TFI algorithm calculates a representative value for each layer thickness parameter from the quasi-stable period after the blink relaxation and before the next blink. In addition, the TFI can recognise from the lipid layer time series a momentary drop of thickness which defines the 'lipid break up', and this information is used to derive the lipid break up time (LBUT) from last blink.

\section{DEVICE'S BASIC PERFORMANCE}

Prior to the study, the device performance and calibration were tested on a mock tear film (glass lens with an artificial two layer tear film). This mock tear film has a similar optical structure to the tear film and cornea surface. The stability (or variability) of the thickness measurements over the tear film mock was found to be at the subnanometre level for both lipid and muco-aqueous mock layers. The absolute measurements of the tear film mock layer thickness by the TFI matched the independent measurements performed using Ellipsometry methods in an independent laboratory. The TFI device design and implementation addresses the anticipated measurement differences between a mock tear film and a real tear film on top of a human eye, such as:

1. The human eye has saccadic motions including micro-saccadic motions, blinks including partial blinks and other phenomena that interfere with fluent measurements. Capturing and analysing these eye behaviours are critical to determine the true tear film dynamics. The TFI detects these phenomena and takes them under consideration during the measurement process. 
2. With each blink, the tear film is reset with subsequent rearrangement of the tear film parameters. Moreover, even between blinks, the tear film layer structure changes due to evaporation, draining and other processes, which are important to concomitantly assess. The TFI non-contact measurement allows (and even requires) regular blinking to preserve the tear film dynamics. In addition, in order to capture the tear film dynamics between blinks, the TFI measures each layer thickness with nanometre accuracy, 10-15 times/s, which allows for a reconstruction of the tear film layer thickness dynamics of both the muco-aqueous and lipid layers.

\section{STUDY DESIGN}

Prospective, pilot study. We aimed to enrol 50 individuals into the study based on our primary outcome of assessing MALT reproducibility. Fifty individuals give us $81 \%$ power to detect a $15 \%$ difference between the measurements. This number of subjects also gives us $90 \%$ power to detect a $15 \%$ difference between our secondary analysis, a comparison of LBUT to TBUT.

\section{RECRUITMENT}

All patients seen in Meir Medical Center ophthalmic outpatient clinic who met criteria were offered recruitment into the study. As this was a pilot study, we included all comers and did not age and gender match as the goal was to evaluate the safety and reproducibility of the TFI.

\section{Inclusion criteria}

Patients with otherwise healthy external ocular anatomy. This included a mix of subjects with and without DE symptoms and signs.

\section{Exclusion criteria}

Patients were excluded if they had chronic ocular surface disease other than idiopathic DE (eg, Stevens-Johnson syndrome, post-burn ocular injury, herpes simplex virus infection, neurotrophic cornea), had any corneal disease (eg, scarring, oedema), used contact lenses, had an active intraocular inflammation or infection, used eye-drops (with 
the exception of artificial tears) or had intraocular surgery or surgical occlusion of the lacrimal puncta within 3 months. Subjects were asked not to use any topical eye drops on the day of their study visit.

\section{ASSESSMENTS}

\section{DEMOGRAPHIC INFORMATION WAS OBTAINED.}

\section{DE ASSESSMENT}

DE symptoms were assessed using the Ocular Surface Disease Index (OSDI). 14 DE signs were assessed in the same room during morning hours at the Meir Medical Center ophthalmic outpatient clinic by one of three independent investigators who were masked to OSDI scores. The order of testing was (1) TBUT (single measurement per eye after a trace of fluorescein was placed in the inferior meniscus off a paper strip), (2) corneal staining (the cornea was divided into four areas and staining was graded in each area on a scale of $0=$ none to $3=$ severe; these four scores were then averaged) and (3) Schrimer's test without anaesthesia (measured at $5 \mathrm{~min}$ ). Subjects were split into three groups based on the presence of DE signs. We opted to group individuals by signs (as opposed to symptoms+signs) as the TFI dynamically captures information on the aqueous and lipid components of tears. There are many factors that contribute to DE symptoms beyond tear components (anatomy, corneal sensitivity, trigeminal pathways beyond the cornea) and as such, we would not expect machine assessed parameters to align with DE symptoms.

Controls (no DE signs) were defined by Schrimer $>10 \mathrm{~mm} / 5 \mathrm{~min}$, TBUT $>7 \mathrm{~s}$ and corneal staining $=0$ in both eyes; mild-moderate $D E$ was defined by one or more of the following: Schirmer between 2 and $10 \mathrm{~mm} / 5 \mathrm{~min}$, TBUT between 1 and $7 \mathrm{~s}$ or corneal staining of 1 or 2; severe DE was defined by one or more of the following: Schirmer $\leq 2 \mathrm{~mm} / 5 \mathrm{~min}$, TBUT $\leq 1 \mathrm{~s}$ or corneal staining=3. We chose to group individuals based on these three DE parameters as they (1) are well-established diagnostic indicators of DE; (2) do not require additional instrumentation to capture beyond that routinely available in an eye clinic and (3) two of the three parameters (TBUT and Schirmer) have TFI-derived metrics which we wished to compare. 


\section{TFI device based on three-dimension tomography white light interference measurement}

TFI measurements were taken 90 min after DE testing to allow sufficient time for stabilisation of the ocular surface. A second measurement was taken an average of 45 min after the first (with a minimum $30 \mathrm{~min}$ ). The high correlation between the first and second TFI measurements reflects that 90 min was a sufficient time for ocular surface stabilisation. Based on the reported high correlation between subject eyes and in order to reduce the risk for the patients, only one eye from each subject were examined. For individuals with DE (mild-moderate or severe DE signs), the more severely affected eye was imaged. For controls, the eye with the healthier clinical tear film parameters was imaged. This was done as DE signs are highly variable, $\frac{15}{}$ and for this pilot study, we wanted to test the ability of the TFI to image a wide range of tear film parameters ranging from severely abnormal to normal. The individual performing the imaging was masked to the results of clinical testing.

The imaging process requires that the patient sit in front of the device with his/her head positioned on the chin rest. The operator aligned the optics to the corneal centre and began recording. The device was manually aligned by a user friendly joystick and data was recorded and saved for analysis. Test time was approximately 40 s/eye. The images and spectral information were then blindly processed using built in software and several output parameters were obtained including: MALT averaged over the measurement window, LLT averaged over the measurement window and LBUT time from last blink to momentary drop in the LLT level. Of note, this pilot study was performed with a prototype device and not all tear film parameters could be calculated from each scan. The technology has been subsequently improved and can now derive the major parameters from more than $90 \%$ of the scans within a couple of minutes.

\section{STATISTICAL ANALYSIS}

All statistical analyses were performed using SPSS V.22.0 statistical package. Frequencies and descriptive statistics were applied to the data, as appropriate. To evaluate the new imaging technology, we first examined safety and then focused on 
reproducibility. Reproducibility was examined via intraclass correlation coefficient (ICC), Pearson correlation and Bland-Altman analysis.

We next evaluated correlations between clinically assessed DE parameters and TFI parameters. Specifically, we compared LBUT (TFI parameter) with TBUT (clinical parameter) and MALT (TFI parameter) with Schirmer score (clinical parameter) using Pearson statistics. We then examined TFI parameters across our clinically determined DE groups using analysis of variance methodologies and evaluated the relationship between TBUT and LBUT using Bland-Altman analysis.

Based on receiver operating characteristic analysis, the TFI identified that a MALT value of $1700 \mathrm{~nm}$ and a LBUT value of $7 \mathrm{~s}$ best distinguished between the DE and healthy populations. Based on these cut-offs, the machine automatically designated individuals to the DE or control group. This machine-generated categorisation was then compared with our clinical grouping to calculate a sensitivity and specificity of a DE diagnosis. Finally, we re-evaluated our findings considering the effect of age, given demographic differences between the groups.

\section{RESULTS}

\section{STUDY POPULATION}

Forty-nine patients participated in the study. The mean age of all participants was 58.8 years (SD 15.9). The majority of subjects were female $(69 \%, n=34)$. Cases with $D E$ were older than their counterparts without DE (table 1). 


\begin{tabular}{|c|c|c|c|c|}
\hline DE categorisation & Healthy $(n=12)$ & $\begin{array}{l}\text { Mild-moderate } \\
(\mathrm{n}=22)\end{array}$ & $\begin{array}{l}\text { Severe } \\
(n=15)\end{array}$ & $P$ value \\
\hline $\begin{array}{l}\text { Age years, mean } \\
\text { (SD) }\end{array}$ & $44.2(14.1)^{*} \dagger$ & $63.3(14.9)$ & $63.9(11.6)$ & 0.001 \\
\hline $\begin{array}{l}\text { Gender, } \% \text { female } \\
\text { (n) }\end{array}$ & $58 \%(7)$ & $77 \%(17)$ & $68 \%(10)$ & 0.5 \\
\hline OSDI, mean (SD) & $1.65(3.9)^{\star} \dagger$ & $32.9(21.5) \ddagger$ & $45.3(20.6)$ & $<0.001$ \\
\hline TBUT, s§, mean (SD) & $13.5(4.8)^{\star} \dagger$ & $4.1(1.5)$ & $2.7(1.5)$ & $<0.001$ \\
\hline $\begin{array}{l}\text { Staining§, mean } \\
\text { (SD) }\end{array}$ & $0(0) \dagger$ & $0.42(0.39) \ddagger$ & $1.1(1.16)$ & $<0.001$ \\
\hline $\begin{array}{l}\text { Schirmer, mm§, } \\
\text { mean (SD) }\end{array}$ & $28.1(8.1)^{\star} \dagger$ & $6.4(5.1)$ & $3.2(4.4)$ & $<0.001$ \\
\hline mean (SD), $\mathbf{n}$ & Healthy & Mild-moderate & Severe & $P$ value \\
\hline $\begin{array}{l}\text { First muco-aqueous } \\
\text { layer thickness, nm }\end{array}$ & $\begin{array}{l}3757(989) \\
\mathrm{n}=10\end{array}$ & $2983(808) n=20$ & $\begin{array}{l}3043(1084) \\
n=14\end{array}$ & 0.099 \\
\hline $\begin{array}{l}\text { Second muco- } \\
\text { aqueous layer } \\
\text { thickness, } \mathrm{nm}(+)\end{array}$ & $\begin{array}{l}4173(1043) \\
n=9\end{array}$ & $2961(827) n=21$ & $\begin{array}{l}3238(1011) \\
n=11\end{array}$ & 0.008 \\
\hline $\begin{array}{l}\text { Lipid break up } \\
\text { time, s }\end{array}$ & $\begin{array}{l}11.2(10.9)^{\star} \dagger \\
n=7\end{array}$ & $3.3(1.7) n=12$ & $2.9(2.2) n=8$ & 0.013 \\
\hline $\begin{array}{l}\text { First lipid layer } \\
\text { thickness, nm }\end{array}$ & $\begin{array}{l}38.8(12.4) \\
\mathrm{n}=11\end{array}$ & $43.4(14.7) n=21$ & $\begin{array}{l}51.9(36.9) \\
n=14\end{array}$ & 0.358 \\
\hline $\begin{array}{l}\text { Second lipid layer } \\
\text { thickness, nm }\end{array}$ & $\begin{array}{l}33.2(10.7) \\
n=12\end{array}$ & $43.9(12.4) n=21$ & $\begin{array}{l}41.4(19.8) \\
n=12\end{array}$ & 0.128 \\
\hline
\end{tabular}

*Significant difference between mild-moderate and severe groups. Significant difference between healthy and mild-moderate groups.

tSignificant difference between healthy and severe groups.

‡Significant difference between mild-moderate and severe groups.

$\S$ Reported signs for the study eye only.

$\mathrm{DE}$, dry eye; OSDI, ocular surface disease index; TBUT, tear break up time.

\section{SAFETY}

No subjects reported discomfort during or after the scanning process. No short-term or long-term adverse effects were noted during the study.

\section{REPRODUCIBILITY}


The first and second MALT measurements were highly correlated with an ICC of 0.938 $(F=16.116, p<0.001)$ and a Pearson coefficient $(r)$ of $0.884\left(R^{2}=0.781, p<0.001\right.$, $\mathrm{n}=39$; figure 1). Bland-Altman analysis demonstrates good $x$-axis distribution and no $y-$ axis bias indicting good agreement between test and retest, with only 5 of 46 subjects exceeding the $2 \sigma$ threshold (figure 2).

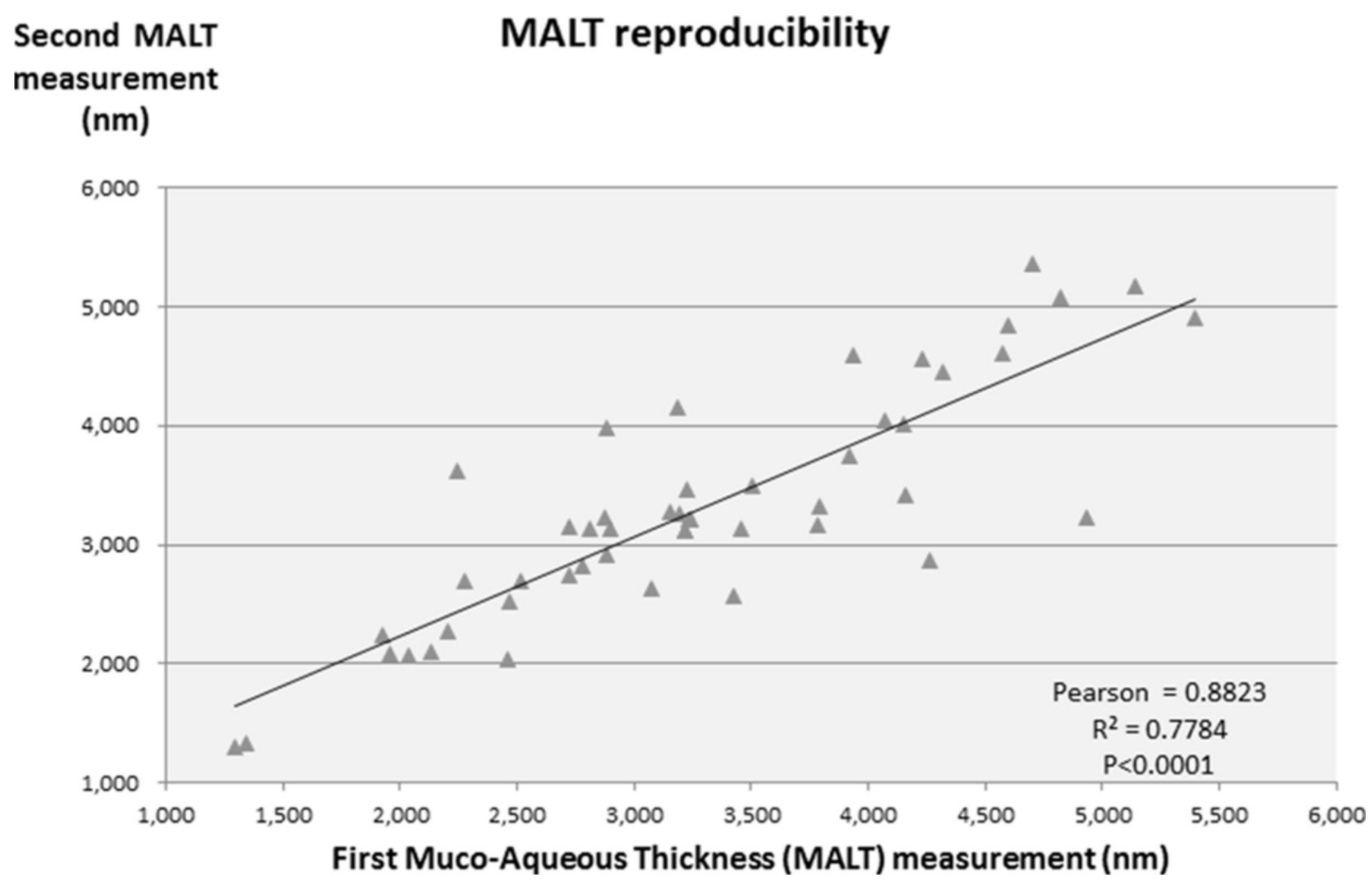

Figure 1

Scatter plot demonstrating excellent reproducibility in muco-aqueous layer measurements with a Pearson coefficient of 0.88 . 


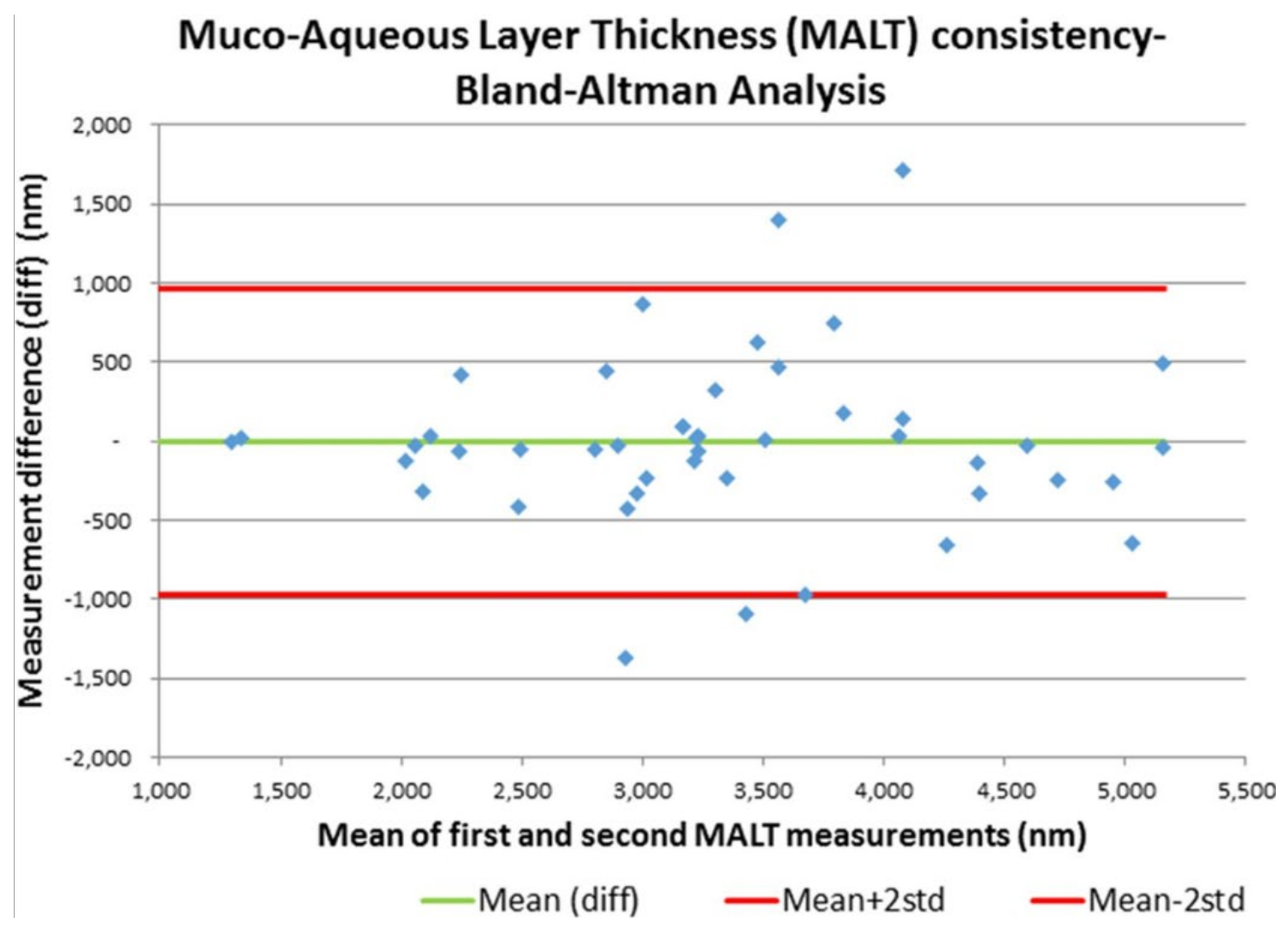

Figure 2

Bland-Altman analysis demonstrating good consistency in muco-aqueous layer measurements.

\section{CORRELATIONS BETWEEN TFI AND CLINICALLY ASSESSED PARAMETERS}

LBUT as measured by the TFI significantly correlated with the clinical measure of TBUT, with an ICC of $0.840(F=6.264, p<0.001)$ and Pearson coefficient $(r)$ of $0.729\left(R^{2}=0.531\right.$, $\mathrm{p}<0.001, \mathrm{n}=27)$. Furthermore, based on a paired-samples t-test comparison, no significant difference was observed between the two measurements, suggesting that the TFI LBUT measurement accurately represents the clinical measure of TBUT (TBUT=6.5 $s ;$ LBUT=5.23 s; $p=0.17 ; n=27$ ).

Bland-Altman analysis further demonstrates good agreement between LBUT and TBUT. The figure 3 demonstrates reasonable $x$-axis distribution reflecting the study population distribution. There is minimal y-axis bias ( $1 \mathrm{~s}$ bias with a SD of $5.5 \mathrm{~s}$ ), indicating that the 
LBUT is on average $1 \mathrm{~s}$ shorter than the TBUT, and only 2 of 46 data points exceeded the $2 \sigma$ threshold.

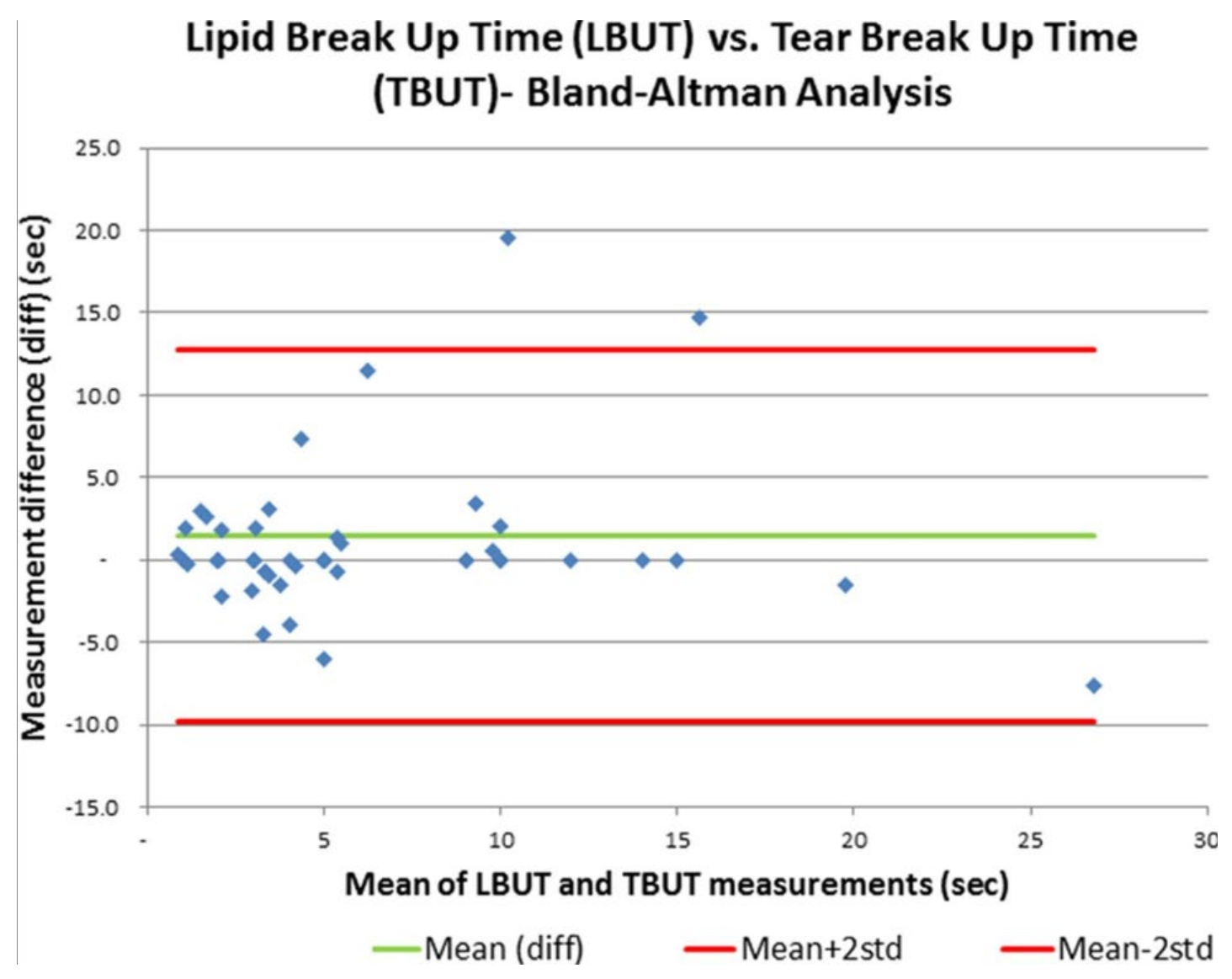

Figure 3

Bland-Altman analysis demonstrating that the lipid break up time closely mirrors the clinically assessed tear break up time

MALT as measured by the TFI significantly correlated with the clinically measured Schirmer score, with a Pearson coefficient $(r)$ of $0.306\left(R^{2}=0.093, p=0.044\right)$ and 0.472 $\left(R^{2}=0.222, p=0.02\right)$ for the first and second MALT measurements, respectively.

\section{TFI parameters by clinical DE groups}

The MALT was thinner in the DE groups (mild-moderate and severe) compared with the control group (table 1). In a similar manner, LBUT as measured by the TFI was significantly faster in the DE groups compared with control group. 
Of note, TFI generated thickness maps of the lipid layer (figure 4) highlight the difficulty in using a single central point to quantify lipid layer thickness (LLT). For example, figure $\underline{4 A}$ is derived from a control subject. One can appreciate a homogenous but thin lipid layer (centre value about $30 \mathrm{~nm}$ ). In contrast, figure 4B is derived from a subject with DE. One can appreciate an uneven map with great differences in LLT ranging between 20 and 120 $\mathrm{nm}$, with a centre thickness of about $50 \mathrm{~nm}$.

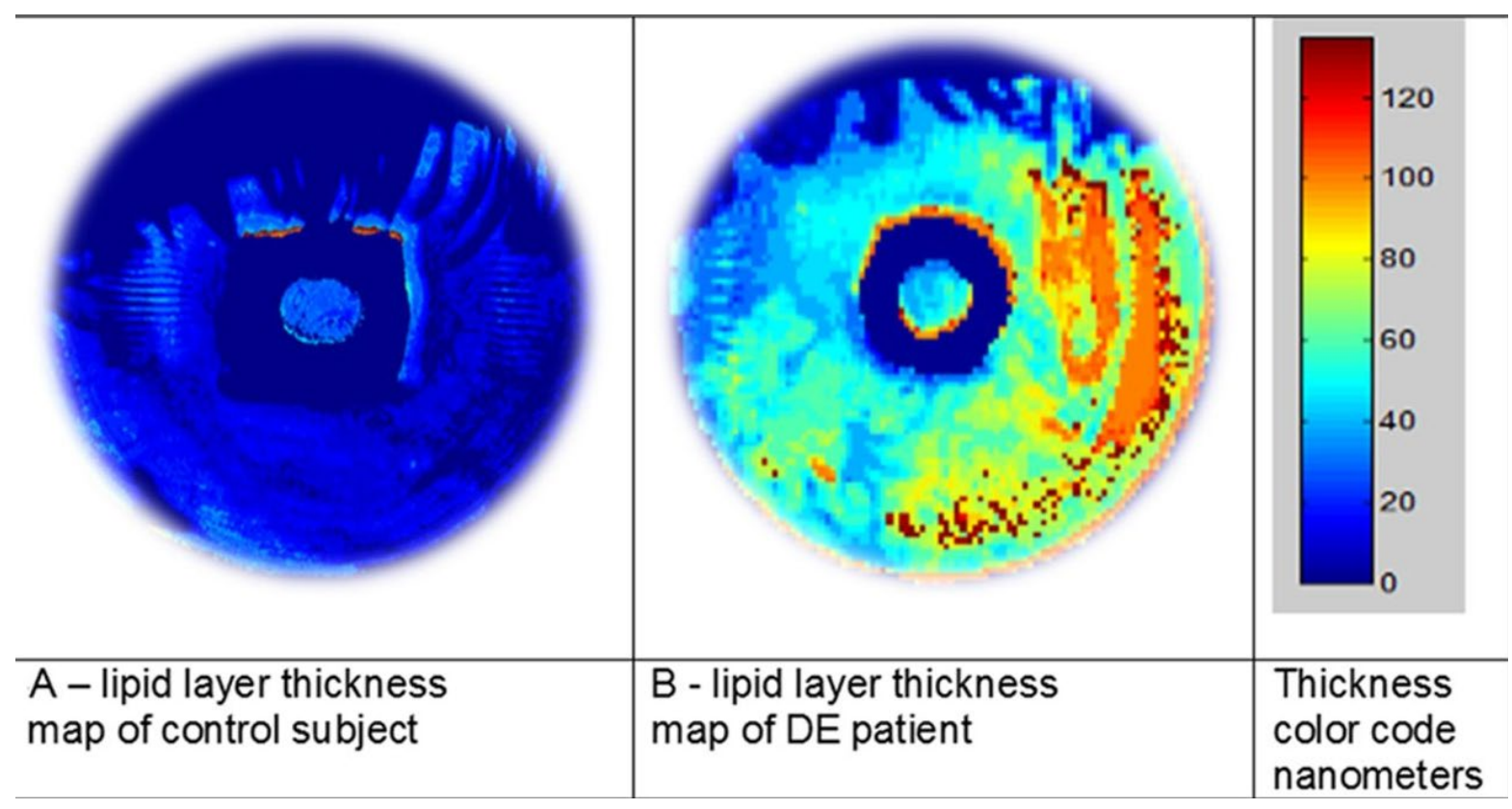

Figure 4

Sample print outs of the lipid layer thickness maps generated from tear film imager scans.

\section{Sensitivity and specificity of the TFI compared with clinical grading}

We utilised thresholding methodology for the TFI parameters which resulted in a machine categorisation of each subject (DE vs no DE). Both MALT and LBUT parameters were used to arrive at this categorisation. We next, compared the machine categorisation to the clinically assessed one. The TFI diagnosed DE with $87 \%$ sensitivity and $88 \%$ specificity in comparison to the clinical DE categorisation, as presented in table 2 . 


\section{Table 2 TFI versus clinical DE classification}

\begin{tabular}{llll}
\hline & \multicolumn{2}{l}{ Signs only } \\
\cline { 3 - 3 } & $\begin{array}{l}\text { Condition } \\
\text { positive (DE) }\end{array}$ & $\begin{array}{l}\text { Condition } \\
\text { negative (no DE) }\end{array}$ \\
\hline \multirow{2}{*}{ TFI $\quad \begin{array}{ll}\text { Condition positive (DE) } \\
\text { Condition negative (no DE) }\end{array}$} & 34 & 1 \\
& 5 & 7 \\
& $\downarrow$ & $\downarrow$ \\
\hline & Sensitivity, 87\% & Specificity, 88\% \\
\hline
\end{tabular}

Positive predictive value: $97 \%$; negative predictive value: $58 \%$.

$\mathrm{DE}$, dry eye; $\mathrm{TFI}$, tear film imager.

\section{Adjustment for comorbidities}

As expected, age significantly correlated with DE signs (Schirmer $r=-0.567$, TBUT $r=-0.539$, corneal staining $r=0.339$ ). Interestingly, age correlated less closely with the TFI parameters of lipid thickness $(r=0.027)$ and MALT (first measurement $r=-0.120$, second measurement $r=-0.158$ ) but not surprisingly, correlated with machine generated LBUT $(r=-0.467)$. In a linear regression model, age did not remain a significant predictor of MALT when considered concomitantly with Schirmer score and of LBUT when considered concomitantly with TBUT.

\section{DISCUSSION}

Our study demonstrates that the TFI is the first imaging modality to reproducibly measure muco-aqueous thickness in human subjects. Although the tear film is highly dynamic and resets with every blink, reproducibility is the basic requirement from any diagnostic method. The average MALT, which is reported by the TFI based on frequent measurements and an averaging algorithm, proved to provide a high level of reproducibility. This is a remarkable achievement, especially for a highly dynamic system like the tear film. No currently available clinical test can capture muco-aqueous thickness, which is a core component of DE. Currently available tests that can be used as surrogates are the Schirmer's test which gives information on tear production and the phenol red thread which gives information on volume. Neither of these, however, directly measures the thickness of the layer on the ocular surface. 
Encouragingly, controls without $D E$ had a thicker muco-aqueous layer compared with individuals with DE. These MALT measurements also correlated well with the clinical assessment of Schirmer score. Additionally, LBUT, as measured by the TFI, was found to be interchangeable with clinically assessed TBUT and was similarly found to be lower in DE versus control subjects. LLT maps further demonstrated the spatial variability in lipid layer in individuals with DE versus without DE. Taken together, our results demonstrate that the TFI parameters are in strong agreement with traditional diagnostic methodologies; yet, the machine offers objective and reproducible tests on new parameters that are not able to be assessed clinically or imaged by currently available technology. Furthermore, the instrument is easy to use, obtains all values within $40 \mathrm{~s}$ and does not require patient contact. In fact, high-quality images were successfully obtained from all subjects enrolled in this study, and none of the subjects reported discomfort or negative effects from imaging. When considering our sensitivity, specificity and predictive values, it is important to consider that there is a lack of a gold standard definition for DE, and so these values change depending on the specific definition. Given the complexity of $\mathrm{DE}$, which has components of aqueous deficiency, lipid deficiency, instability, abnormalities in tear components (inflammation, osmolarity), it is not surprising that objective tests overall have a low sensitivity. Considering this reality, the TFI numbers are better than traditional DE tests that only take into account one component of the tear film ${ }^{4}$ but are still limited by the diagnostic challenged inherent in DE. Similar caution must be applied when examining the machine-generated threshold values used to assess sensitivity. This study was not powered to determine threshold definitions in DE versus control and as such, future studies in expanded populations are needed to evaluate appropriate thresholds for differentiating between health and disease.

The TFI provides several advantages over other clinical and machine-generated DE measures. The TFI allows for an unbiased, non-invasive, quick and dynamic assessment of the muco-aqueous and lipid layers of the tear film. As such, it is more standardised and quicker than the clinical examination and captures information not detected at the slitlamp examination (MALT and LLT). Its nanometre resolution is also superior to currently available imaging devices. To compare, the LipiView can measure the lipid layer at approximately a $30 \mathrm{~nm}$ accuracy. HR-OCT has an axial resolution on the order of 2-5 
$\mu \mathrm{m}^{\mathrm{z}}$ and a measurement rate on the scale of a seconds. $\frac{89}{}$ In terms of dynamics, a limitation of OCT is that even though the A-scan component can scan thousands of images per second, the B-scan component is much slower. Even in a sparse mode, it takes the B-scan about $1 \mathrm{~s}$ to cover a few $15 \mu \mathrm{m}$ lines $3 \mathrm{~mm}$ in length. This seconds long scanning limitation precludes the use of HR-OCT to track tear film dynamics between blinks. The Keratograph measures tear meniscus height and non-invasive TBUT but not quantify tear component thickness.

Our encouraging findings need to be considered, given the limitations of this pilot study which included a small, defined patient population. Furthermore, while the TFI images the central $6 \mathrm{~mm}$ of the cornea, which is the area most central to vision, the exclusion of peripheral cornea limits the evaluation of tear meniscus parameters and the conjunctival regions. Likewise, the TFI does not measure other components of $D E$, such as osmolarity and meibomian gland anatomy. Future studies will need to validate our findings in other DE and non-DE age-matched and gender-matched populations and compare our results with other DE imaging devices such as the Lipiview.

Despite these limitations, the TFI is the first machine that can directly and dynamically measure the MALT, while simultaneously providing information on lipid layer stability. Further studies are needed to address questions regarding the impact of demographics, intereye differences, manifestations of DE subgroups (aqueous vs evaporative deficiency) and effects of contact lens and surgery on tear film parameters. The utility of this instrument for these indications is an important direction of future study.

\section{REFERENCES}

1. Stapleton F, Alves M , Bunya VY, et al . TFOS DEWS II epidemiology report. Ocul Surf2017;15:334-65.doi:10.1016/j.jtos.2017.05.003. 
2. Craig JP , Nichols KK , Akpek EK, et al . TFOS DEWS II definition and classification report. Ocul Surf2017;15:276-83.doi:10.1016/j.jtos.2017.05.008

3. Pouyeh B , Viteri E, Feuer W, et al . Impact of ocular surface symptoms on quality of life in a United States Veterans Affairs population. Am J Ophthalmol2012;153:10616.doi:10.1016/j.ajo.2011.11.030

4. Wolffsohn JS, Arita R, Chalmers R, et al. TFOS DEWS II diagnostic methodology report. Ocul Surf2017;15:539-74.doi:10.1016/j.jtos.2017.05.001

5. Jung JW , Park SY, Kim JS, et al. Analysis of factors associated with the tear film lipid layer thickness in normal eyes and patients with dry eye syndrome. Invest Ophthalmol Vis Sci2016;57:4076-83.doi:10.1167/iovs.16-19251

6. Cox SM, Nichols KK, Nichols JJ . Agreement between automated and traditional measures of tear film breakup. Optom Vis Sci2015;92:e25763.doi:10.1097/OPX.0000000000000648

7. Cui $L$, Wang J , Perez $V L$, et al . Visualization of the precorneal tear film using ultrahigh resolution optical coherence tomography in dry eye. Eye Contact Lens2012;38:2404.doi:10.1097/ICL.0b013e318257a108

8. Napoli PE, Coronella F, Satta GM, et al . A simple novel technique of infrared Meibography by means of spectral-domain optical coherence tomography: a crosssectional clinical study. PLoS One2016;11:e0165558.doi:10.1371/journal.pone.0165558

9. Napoli PE , Coronella F, Satta GM , et al . Evaluation of the adhesive properties of the cornea by means of optical coherence tomography in patients with meibomian gland dysfunction and lacrimal tear deficiency. PLoS One2014;9:e115762.doi:10.1371/journal.pone.0115762

10. King-Smith PE , Fink BA, Fogt $\mathrm{N}$, et al . The thickness of the human precorneal tear film: evidence from reflection spectra. Invest Ophthalmol Vis Sci2000;41:3348-59.

11. Nichols JJ , Mitchell GL , King-Smith PE. Thinning rate of the precorneal and prelens tear films. Invest Ophthalmol Vis Sci2005;46:2353-61.doi:10.1167/iovs.05-0094

12. King-Smith PE, Hinel EA, Nichols JJ . Application of a novel interferometric method to investigate the relation between lipid layer thickness and tear film thinning. Invest Ophthalmol Vis Sci2010;51:2418-23.doi:10.1167/iovs.09-4387 
13. Bron AJ , de Paiva CS , Chauhan SK, et al . TFOS DEWS II pathophysiology report. Ocul Surf2017;15:438-510.doi:10.1016/j.jtos.2017.05.011

14. Schiffman RM , Christianson MD , Jacobsen G , et al . Reliability and validity of the ocular surface disease index. Arch Ophthalmol2000;118:61521.doi:10.1001/archopht.118.5.615

15. Bron AJ , Tomlinson A, Foulks GN , et al . Rethinking dry eye disease: a perspective on clinical implications. Ocul Surf2014;12(2 Suppl):S1-S31.doi:10.1016/j.jtos.2014.02.002 